\title{
Design of a 10-bit 100 MSamples/s BiCMOS D/A converter
}

\author{
Jørgensen, Ivan Harald Holger; Tunheim, S. A.
}

Published in:

Circuits and Systems, 1995., Proceedings., Proceedings of the 38th Midwest Symposium on

Link to article, DOI:

10.1109/MWSCAS.1995.510193

Publication date:

1995

Document Version

Publisher's PDF, also known as Version of record

Link back to DTU Orbit

Citation (APA):

Jørgensen, I. H. H., \& Tunheim, S. A. (1995). Design of a 10-bit 100 MSamples/s BiCMOS D/A converter. In Circuits and Systems, 1995., Proceedings., Proceedings of the 38th Midwest Symposium on (Vol. 2, pp. 730733). IEEE. https://doi.org/10.1109/MWSCAS.1995.510193

\section{General rights}

Copyright and moral rights for the publications made accessible in the public portal are retained by the authors and/or other copyright owners and it is a condition of accessing publications that users recognise and abide by the legal requirements associated with these rights.

- Users may download and print one copy of any publication from the public portal for the purpose of private study or research.

- You may not further distribute the material or use it for any profit-making activity or commercial gain

- You may freely distribute the URL identifying the publication in the public portal 


\title{
Design of a 10-bit 100 MSamples/s BiCMOS D/A Converter
}

\author{
Ivan Harald Holger Jørgensen ${ }^{1}$ and Svein Anders Tunheim ${ }^{2}$ \\ ${ }^{1}$ Electronics Institute, Building 349, \\ Technical University of Denmark (DTU), DK-2800 Lyngby, Denmark \\ Phone: (+45) 459342 07, Fax: (+45) 458801 17, E-mail: ihhj@ei.dtu.dk \\ ${ }^{2}$ SINTEF, P.O.Box 124 Blindern, N-0314 OSLO, Norway. \\ Phone: (+47) 220673 00, Fax: (+47) 220673 50, E-mail: Svein.Anders.Tunheim@si.sintef.no
}

\begin{abstract}
A 10-bit 100MSamples/s current-steering D/A converter (DAC) has been designed and processed in a $0.8 \mu \mathrm{m}$ BiCMOS process. The DAC is intended for applications using direct digital synthesis, and focus has been set on achieving a high spurious free dynamic range (SFDR ${ }^{1}$ ). The main part of the DAC is a matrix of current cells. To reduce skew between the steering signals to the current cells, an emitter-coupled logic (ECL) flip-flop, clocked by a global ECL clock, is included in each current cell. A bipolar differential pair, steered by the differential output of the ECL flip-flop, is used in each current cell to steer the current. At a generated frequency of $f_{g} \approx 0.3 \cdot f_{s}\left(f_{s}=100 \mathrm{MSam}\right.$ ples $/ s$ ), the simulated SFDR is larger than $60 \mathrm{~dB}$. The DAC operates at $5 \mathrm{~V}$, and has a power consumption of approximately $650 \mathrm{~mW}$. The area of the chip-core is $2.2 \mathrm{~mm} \times 2.2 \mathrm{~mm}$. Furthermore a measure to estimate the SFDR for the DAC based on short term simulations is presented. This measure seems to correspond very well with SFDR for long term simulations.
\end{abstract}

\section{INTRODUCTION}

High-speed and high resolution DACs are used in various applications such as direct digital synthesis (DDS), arbitrary waveform generation (AFG), new TV/Video systems and communications local oscillators. For these applications the dynamic performance of the DAC is very critical. For the DAC described in this paper, focus has been set on achieving a high spurious free dynamic range (SFDR) at high generated frequencies, $f_{g}$, compared to the conversion rate, $f_{s}$ (100MSamples $/ s$ ), i.e., $f_{g} \approx 0.3 \cdot f_{s}$. Any static or dynamic nonlinearity will generate spurious frequencies at the DAC output. Static nonlinearities can be reduced significantly by careful layout and by using different kinds of switching schemes for the current cells [5], [6]. The main dynamic nonlinearities are nonequal and code dependent rise and fall times, delay between rise and fall transients, and nonlinear clock and data feedthrough. For a typical current steering $\mathrm{DAC}$ one of the main challenges is to generate steering signals for the current cells with very little skew between these

\footnotetext{
${ }^{1}$ SFDR is measured from the generated frequency to the highest harmonic or non-harmonic spur within the frequency band $0-f_{s} / 2$.
}

[1], [3], [5]. A solution is to use clocked current cells. One then has to generate a global clock for the current cells that has an acceptable low clock skew over the current cell matrix. This is an advantage because clock-skew can more easily be controlled than the delays of different steering signals.

The DAC presented in this paper is constructed using this scheme. Each current cell contains an ECL flip-flop. The skew between the steering signals for the different current cells are then controlled by a global clock signal.

The DAC architecture is described in section II, and the current cells are described in section III. Section IV.A shows the simulation results of the DAC with special emphasis on SFDR. A measure, especially useful during circuit simulations of the dynamic performance of high-speed DACs, is proposed in section IV.B.

\section{DAC ARCHITECTURE}

The DAC presented in this paper is based on the well-known current steering principle [1]-[7]. The DAC architecture is shown in Fig. 1.

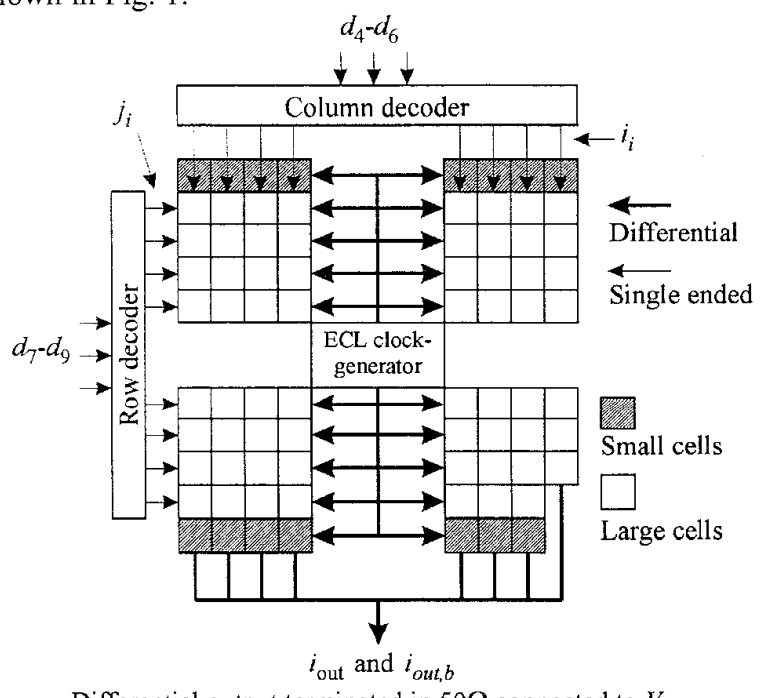

Differential output terminated in $50 \Omega$ connected to $V_{d d}$

Fig. 1. DAC Architecture 
The current cells are arranged in a matrix. This matrix consists of 63 large current cells that determine the 6 most significant bits (6 MSB), and 15 smaller cells that determine the 4 least significant bits ( 4 LSB). One large current cell sinks a current 16 times the current in a small current cell. The DAC operates as follows: The digital input word, $d_{9}$ (MSB)$d_{0}(\mathrm{LSB})$, is stored in an input register, and decoded into row and column signals (for the $6 \mathrm{MSB}$ ), and then latched before being fed to the current cells. In Fig. 1 the registers and latches are contained in the column and row decoders. The decoding ensures that a minimum of current cells are turned on or off; this reduces dynamic nonlinearities. The hierarchical switching scheme [6] is used for the large current cells to reduce nonlinearities. This switching scheme reduces both symmetrical and graded errors. For the small current cells, a simple "chessboard scheme" is used controlled by the digital signals $d_{3}-d_{0}$.

In each current cell (see Fig. 2) the CMOS steering signals, $i, i+1$ and $j$, are decoded by CMOS logic. The decoded signal is then converted into ECL levels, $v_{i n}$ and $v_{i n, b}$, and latched by an ECL flip-flop clocked by the global differential ECL clock. Thus, one reduces the problem with skew between the steering signals over the matrix. The differential current outputs of all current cells are connected to the DAC output pins $i_{\text {out }}$ and $i_{\text {out }, b}$ as shown in Fig. 1. These are, externally, each connected to a $50 \Omega$ resistor that again is connected to the positive power supply $(5 \mathrm{~V})$. The maximum output current from the DAC is $20 \mathrm{~mA}$. The ECL clock generator is placed in the middle of the current cell matrix to minimise clock skew. This also causes the clock skew to be a symmetrical error. Therefore it will, to some extent, be reduced by the hierarchical switching scheme.

The DAC consumes approximately $650 \mathrm{~mW}$. The temperature operating range for the $\mathrm{DAC}$ is from $-40^{\circ} \mathrm{C}$ to $80^{\circ} \mathrm{C}$.

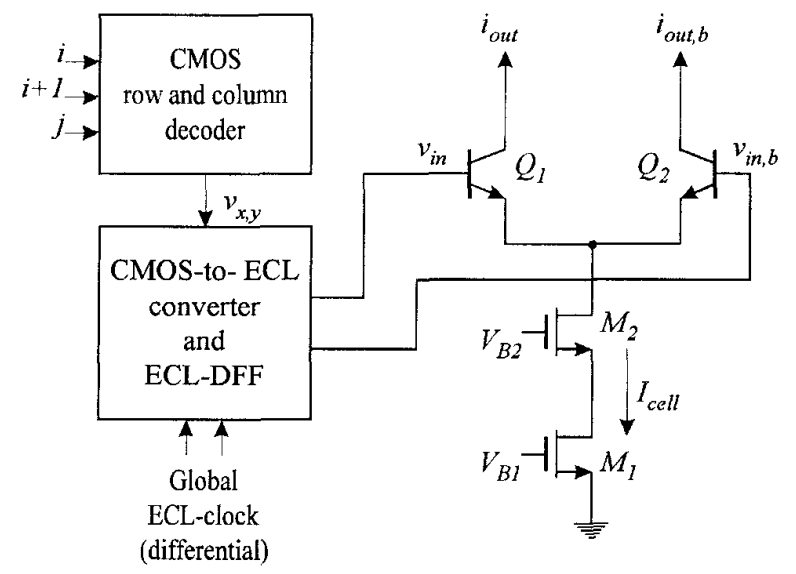

Fig. 2. The large current cell

\section{Current Cells}

A schematic of the large current cells is shown in Fig. 2 [2]. A differential pair with bipolar transistors, $Q_{1}$ and $Q_{2}$, is used to steer the current. The cascode current sink consists of two NMOS transistors $M_{1}$ and $M_{2}$. The current cell includes a CMOS input section which decodes the row and column signals. The decoded signal is converted into a differential ECL signal that is latched before fed to the differential pair, $Q_{1}$ and $Q_{2}$.

The use of a bipolar differential pair offers several advantages compared to the MOS counterpart. As the DAC should operate at a very high conversion rate, it is obvious to use fast bipolar transistors to toggle the current $I_{\text {cell }}$ between the two outputs. Furthermore, the required voltage swing at the input, $v_{i n}-v_{i n, b}$, is much lower compared to the input swing of a MOS differential pair in a practical design. The differential input voltage that guaranties that the total erroneous output current from all $N_{\text {cells }}$ current cells is less than $1 / 2 \mathrm{LSB}$ of the total current, is given by (1). In (1) the collector currents $I_{C 1}$ and $I_{C 2}$ are given by the well-known exponential equation for the bipolar transistor. $V_{T}$ is the thermal voltage which equals $26 \mathrm{mV}$ at room temperature.

$$
\frac{I_{C 1}}{I_{C 2}}>2^{N+1} N_{\text {cells }} \Rightarrow \Delta v_{\text {in }}=v_{\text {in }}-v_{\text {in, }}>V_{T} \ln \left(2^{N+1} N_{\text {cells }}\right)
$$

Using the number of bits $N=10, N_{\text {cells }}=64$ (63 large, plus 15 small which equals approximately 1 large) we find that $\Delta v_{b e}>12 V_{T}=312 \mathrm{mV}$. This small ECL voltage swing and the fast ECL flip-flop output signals potentially give low spurious frequencies at the DAC output.

The delay of the output from the CMOS input section, $v_{x, y}$, in the current cell varies strongly depending on the input signals $i, i+1$ and $j$. The delay, rise and fall times for $v_{x, y}$ vary strongly dependent on the inputs $i, i+1, j$. If these CMOS signals, $v_{x, y}$, were used to steer the current cells directly the output signal contain higher level of spurious frequencies. The output from the CMOS part is therefore latched in the ECL section. The skew between the steering signals is therefore only dependent on the clock skew in the global ECL clock. The worst case clock skew over the matrix is designed to be less than $8 \mathrm{ps}$.

The small current cells have the same architecture as the large ones but $i$ is connected to $V_{d d}, i+1$ is connected to ground and the cells are only steered by $j$.

Because of $\alpha$-mismatch, the bipolar differential pairs introduce current mismatch between the current cells. Assuming a $\Delta \beta / \beta$ better than $2 \%$ for transistors on the same chip, one obtain an $\Delta \alpha / \alpha$ of approximately $0.02 \%$. This will be of minor importance compared to the other mismatch sources, such as ground potential variations and mismatch between NMOS current sinks. 


\section{DAC TOP-LEVEL SIMULATIONS}

\section{A. Simulation of SFDR}

The DAC has been simulated with Accusim from Mentor Graphics. To simulate the SFDR, an ideal sine wave was forced to an ideal A/D converter, to produce a pure 10-bit digital "sine" that was used as input to the DAC. Fig. 3 shows a segment of the resulting DAC output. Here, the generated frequency was approximately $30 \mathrm{MHz}$, and the conversion rate was $100 \mathrm{MSamples} / \mathrm{s}$. During this simulation, a lumped resistance model of the ground network, and a lumped resistance and capacitance model of the ECL clock tree, were used. About 75 periods of the generated frequency were simulated. Fig. 4 shows the FFT of the differential DAC output signal shown in Fig. 3. (window function: Kaiser-Bessel). The simulation shows that SFDR $>60 \mathrm{~dB}$.

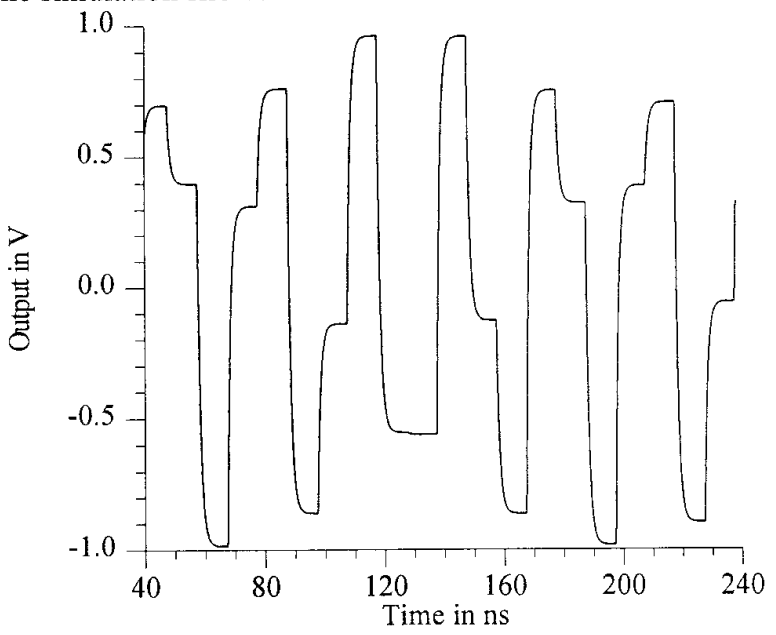

Fig. 3. Simulated differential DAC output, $v_{\text {out }}(t)-v_{\text {out }, b}(t)$, $f_{g} \approx 0.3 \cdot f_{s}, f_{s}=100$ MSamples $/ s$

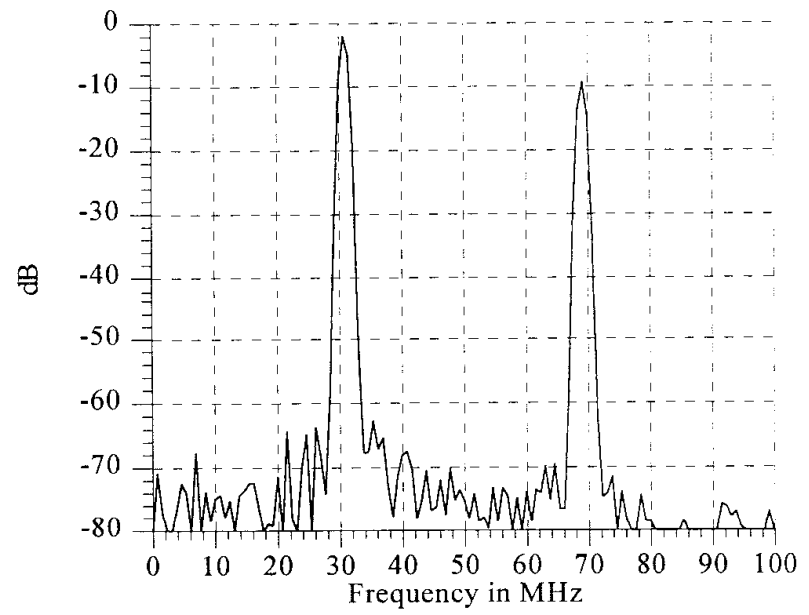

Fig. 4. FFT of the simulated DAC output, $f_{g} \approx 0.3 \cdot f_{s}$, $f_{s}=100 \mathrm{MSamples} / \mathrm{s}$

\section{B. A Measure of DAC Dynamic Performance}

Top level simulations of the DAC to evaluate the SFDR are very time consuming as one has to simulate several periods of the generated frequency. E.g., the simulation of the final DAC that produced the result in Fig. 4 lasted for approximately 2 weeks on an HP 735 workstation.

A measure, mean error energy per conversion period $E_{\text {mean }}$, that estimates the SFDR of the final DAC, is therefore proposed. The measure is intended to be used for DACs with a switching scheme like the one used in this paper, i.e., a switching scheme that guaranties that a minimum of current cells are turned on or off each clock period. This measure is based on the dynamic behaviour of the entire DAC during a full scale output transient (input codes: $00 . .00 \rightarrow 11 . .11$ ). The simulation time is therefore reduced significantly.

For a DAC with differential output, $v_{\text {out }}(t)$ and $v_{\text {out }, b}(t)$, one first finds the error voltage for a full swing transient:

$$
v_{\text {error }}(t)=v_{\text {out }}(t)+v_{o u t, b}(t)-v_{D C}
$$

$v_{D C}$ is the mean value of $v_{\text {out }}(t)+v_{\text {out }, b}(t) . v_{\text {error }}(t)$ will include the effects of different rise and fall times, different delays for rise and fall transients, etc. Ideally $v_{\text {error }}(t)$ should equal zero. The error energy per conversion period, $T_{C}$, is then calculated:

$$
E_{\text {error }}=\int_{0}^{T_{c}}\left|v_{\text {error }}(t)\right| d t \quad[V \cdot s]
$$

To estimate $E_{\text {mean }}$ for a given generated frequency, one has to multiply $E_{\text {error }}$ with the average step size at the DAC output. For a DAC generating a sine wave, the relative average step size at the output, $S_{\text {mean }}$, compared to the full ouput swing of the DAC, is given by:

$$
S_{\text {mean }} \approx 2 \frac{f_{g}}{f_{s}}
$$

In (4) it is assumed that the digital "sine" utilises the full output swing of the DAC. One can now calculate $E_{\text {mean }}$ at the DAC output:

$$
E_{\text {mean }} \approx S_{\text {mean }} \cdot E_{\text {error }} \quad[V \cdot s]
$$

This energy is compared to the energy of one LSB at the output of the DAC which is given by

$$
E_{L S B}=V_{\text {full-swing }} T_{C} 2^{-N}
$$

Here $V_{\text {full-swing }}$ is the maximum differential voltage swing at the output of the DAC. As a conservative design target $E_{\text {mean }}$ should be less than $E_{L S B} / 2$ if the SFDR should be higher than $N \cdot 6 d B$.

$E_{\text {error }}$ was simulated to $17.8 \mathrm{p} V \mathrm{~s}\left(T_{C}=10 \mathrm{~ns}\right)$. This means that $E_{\text {mean }} \approx 10.7 p V s$ for a generated frequency of $0.3 \cdot f_{s}$. Comparing $E_{\text {mean }}$ to $E_{L S B} / 2$, which equals $10 p V s$, one finds that this corresponds to SFDR $\approx 60 d B$ that also was the result in section IV.A. The actual $E_{\text {mean }}$ for the simulation shown in 
Fig. 3 and Fig. 4 was $11.2 p V s$ which corresponds very well with the estimated value of $E_{\text {mean }}$.

This measure offers the opportunity to compare the performance of different current cells in a DAC without performing long term top level simulations. The dynamic performance of a DAC is often given in terms of the glitch energy. Contrary to $E_{\text {mean }}$, the glitch energy is not based on a full scale transition. The full scale transition includes all nonideal dynamic effects. Therefore $E_{\text {mean }}$ provides a better estimate of the dynamic performance than the conventional glitch energy which only contains the nonideal effects of one or more specific transients. Also some DAC architectures, such as the one presented here, inherently do not have the typical large glitch energy at the transition $011 \ldots 11$ to 100..00. Therefore $E_{\text {mean }}$ is a more appropriate measure.

$E_{\text {mean }}$ was also used to evaluate the dynamic behaviour of a single current cell. However, $E_{\text {mean }}$ multiplied with the number of current cells gives a too optimistic estimate of the dynamic behaviour of the entire DAC. The measure still applies very well when comparing the dynamic behaviour of different current cells.

\section{LAYOUT CONSIDERATIONS}

The DAC is processed in a $0.8 \mu \mathrm{m}$ double-metal double-poly BiCMOS process provided by Austria Mikro Systeme International GmbH (AMS). To minimise switching noise from the CMOS- and ECL-logic to couple to the output of the DAC, the power supplies for the CMOS- and ECL-logic and the current sinks are routed separately. The current $I_{\text {cell }}$ from each large current cell introduce a voltage drop in the analogue ground. The analogue ground wires are therefore made wide. Also, several pads on each side of the current cell matrix are used to connect the analogue ground. Furthermore, all different parts of the circuitry are surrounded by guardrings and all major parts by double guardrings to minimise noise injected to the output through the substrate. A separate power pad has been used for the n-well parts of the guard rings. All bias lines, both to the ECL-logic and the current sinks in the current cells, have both local and global decoupling capacitors to minimise spikes on these. Horizontal and vertical shielding between the analogue routing and digital data lines (both CMOS and ECL) have been used to minimise coupling between these. Careful layout and design were necessary to obtain sufficient time margin for the data setup with respect to the ECL clock.

Clock skew, ground potential variation, etc., that generate spurious frequencies at the DAC output have been taken into account and were designed to introduce errors at the output, each less than $1 / 2$ LSB. The switching scheme will tend to cancel must of them. The total error at the output is therefore not the sum of all errors. The size of the chip-core is $2.2 \mathrm{~mm}$ $\times 2.2 \mathrm{~mm}$.

\section{EXPERIMENTAL RESULTS}

At the present time no measurement results are available as the chip is currently being processed.

\section{Conclusion}

A 10-bit 100MSamples/s current steering DAC has been designed and fabricated in a $0.8 \mu \mathrm{m}$ BiCMOS process. The core of the DAC consists of a matrix of current cells. Each current cell includes CMOS decoding logic, a CMOS-to-ECL converter, an ECL flip-flop and a bipolar differential pair to steer the current. The ECL flip-flop is clocked by a global ECL differential clock. The differential output of the flip-flop directly steers the bipolar differential pair. The DAC shows a considerable potential for achieving a high SFDR. A measure to predict the SFDR from short term simulations is presented, and it seems to correspond very well with the actual simulated SFDR.

\section{ACKNOWLEDGEMENTS}

The authors thank their colleagues at the ASIC group at SINTEF Instrumentation, especially Geir Førre, for helpful and inspiring discussions. Furthermore, the authors thank Mustapha Slimane Kadi for valuable help. This work has been carried out under contract of EU under ESPRIT contract 8795 (AMFIS). SINTEF's work has been funded by the Research Council of Norway. Ivan H. H. Jørgensen acknowledges the Ph.D. scholarship granted by the Danish Technical Research Council.

\section{REFERENCES}

[1] Geir Førre, Svein Anders Tunheim, Jakob Gakkestad and Arne Kjensmo, "Design, Simulation and Layout of a $100 \mathrm{Msamples} / \mathrm{s}$ CMOS DAC", SINTEF Report no. STF31 F93034, September 1993.

[2] Ivan Harald Holger Jøgensen, "BiCMOS Current Cell with Cascoded CMOS Current Sink", Internal SINTEF Memo, November 1994 .

[3] S.Chin and C.Wu, "A 10-b 125MHz CMOS Digital-to-Analog Converter (DAC) with Threshold-Voltage Compensated Current Sources", IEEE JSSC, vol. 29, no. 11, November 1994.

[4] B. G. Henriques and José E. Franca, "A High-Speed Programmable CMOS Interface System Combining D/A Conversion and FIR filtering", IEEE JSSC, vol. 29, no. 8, August 1994.

[5] T.Wu et. al, "A Low Glitch 10-bit 75-MHz CMOS Video D/A Converter", IEEE JSSC, vol. 30, no. 1, January 1995.

[6] Y.Nakamura et al., "A 10-b 70MS/s CMOS D/A-converter", IEEE JSSC vol. 26, no. 4, pp. 637-642, Apr. 1991.

[7] C.Bastiaansen et al., "A 10-b 40-MHz 0.8 $\mu \mathrm{m}$ CMOS Current-Output D/A-converter", IEEE JSSC, vol. 26, no. 7, July 1991.

[8] Sverre Dale Moen and Svein Anders Tunheim, "High Level Simulations of a 100Msamples/s DAC", SINTEF Report no. STF31 F94044, October 1994. 